\title{
Designing with Mobile Digital Storytelling in Rural Africa
}

\author{
Nicola J Bidwell ${ }^{12}$ \\ ${ }^{1}$ James Cook University \\ Sch. of Business, Cairns campus \\ Australia \\ nic.bidwell@gmail.com \\ Thomas Reitmaier * ${ }^{2}$ Gary Marsden ${ }^{2}$ \\ ${ }^{2}$ University of Cape Town \\ Susan Hansen \\ Centre for ICT4D \\ South Africa \\ thomas.reitmaier@gmail.com \\ University of Technology Sydney \\ Interaction Design \& Human \\ Practice Lab \& CSIRO, ICT Centre. \\ Australia \\ * Note: Reitmaier is joint-first author with Bidwell. Please abbreviate citation to Bidwell \& Reitmaier et al.
}

\begin{abstract}
We reflect on activities to design a mobile application to enable rural people in South Africa's Eastern Cape to record and share their stories, which have implications for 'cross-cultural design,' and the wider use of stories in design. We based our initial concept for generating stories with audio and photos on cell-phones on a scenario informed by abstracting from digital storytelling projects globally and our personal experience. But insights from ethnography, and technology experiments involving storytelling, in a rural village led us to query our grounding assumptions and usability criteria. So, we implemented a method using cell-phones to localise storytelling, involve rural users and probe ways to incorporate visual and audio media. Products from this method helped us to generate design ideas for our current prototype which offers great flexibility. Thus we present a new way to depict stories digitally and a process for improving such software.
\end{abstract}

\section{Author Keywords}

digital storytelling, mobile devices, oral knowledge, rural, ICT4D, cross-cultural, dialogical approach to design

\section{ACM Classification Keywords}

H5.m. Information interfaces and presentation (e.g., HCI): Miscellaneous.

\section{General Terms}

Design, Experimentation, Human Factors

\section{INTRODUCTION}

Across history, around the world, people have told stories; and adapted the telling of those stories to different media. Writing and technologies to record sound and image enable us to share stories across distance and time; they assist us in drawing those far away into our experience or imagination. At the same time our exposure to stories in media, from comics to soap operas, affects how we create, tell and listen to stories. This, in turn, shapes our use of stories in HCI to depict requirements and engage with users or design software for Digital Storytelling [1] (e.g. to edit video or

Permission to make digital or hard copies of all or part of this work for personal or classroom use is granted without fee provided that copies are not made or distributed for profit or commercial advantage and that copies bear this notice and the full citation on the first page. To copy otherwise, or republish, to post on servers or to redistribute to lists, requires prior specific permission and/or a fee.

CHI 2010, April 10-15, 2010, Atlanta, Georgia, USA.

Copyright 2010 ACM 978-1-60558-929-9/10/04...\$10.00. link images to written text or voice-overs). Relations between media and storytelling contribute dilemmas in designing for communities that rely on direct, unmediated face-to-face communication or a more 'primary orality' [16], due to their antecedents or sparse use of technology. That is, we encounter a paradox in responding to "the exclusion of 'ordinary' people's stories in broadcast media" [10] beyond the digitally saturated world.

In this paper we reflect on our activities to design a mobile digital story application to suit the functions and qualities of storytelling in a rural community in a Xhosa tribal region of South Africa's Eastern Cape. Our goal was to enable users without access to personal computers to preserve, reflect on and share their own life experiences and express their imagination digitally. This implicitly raised the challenge of understanding the local activity of storytelling through the process of design; and, so we advanced this goal by framing design dialogically [20]. That is, we embraced the idea that the meanings we make about storytelling are always unfinalized as they live in sets of relationships between ourselves, others and diverse aspects of settings. Our experience sensitized us to ways that our own interactions with stories arbitrate how we align understandings about ourselves with our users. Thus, here we also aim to show implications of a dialogical approach that are vital in 'crosscultural design', beyond specific digital story software.

We scoped the concept of digital storytelling on mobile platforms using an interaction scenario informed by digital storytelling projects globally and own experience of storytelling. However, insights from technology experiments involving storytelling and ethnography in a remote village led us to query our assumptions about storytelling and usability criteria for mobile digital story software. Our experience yielded nuances in relationships between stories, orality, visual cues and values and insight into their consequences for design. To motivate our account we first outline the potential value of mobile digital storytelling to rural communities and draw attention to challenges in designing for oral traditions that differ from our own. Then we summarise those formative endeavours that drove us to seek to localise storytelling and use a basic technology probe in a method to involve users in exploring ways to incorporate visual and audio media. Next we describe how insights generated by activities with this probe informed features of our current prototype reflect and compare its 
functionality to similar software. Finally, we note relations between ourselves, users and activities that affect our views on storytelling and our ongoing use of our prototype.

\section{Mobile Digital Stories in Rural Development Contexts}

Digital storytelling applications offer unique value in enabling rural communities in developing regions to share local information and participate in decisions affecting their lives [12]. Audio recordings, supported by images, can share information that is unsuited to text and between those who are not literate in their local language. For example, in India rural people have authored, exchanged and uploaded stories to a touch-screen, using non-textual interfaces to Nokia camera-phones [12]. Mobile devices, in particular, can help to situate stories by enabling people to record and share events as they happen during activities, and in places, that are unsuited to computers (e.g. because one cannot hold a lap-top while milking a cow in a field). Storytelling via mobile interfaces can offer access to varied information (e.g. on health, advertising, self-help [12]) and is especially useful for tacit or performed knowledge that rural people routinely transfer informally but is not easily abstracted [3], as they can ascribe meaning by referring to context. The phone's accessibility may also afford a role in citizen media and, together with digital storytelling, enable those with limited influence to raise awareness of their concerns.

\section{Whose Digital Story is it Anyway?}

Before leaping to endorse the potential of the digital story to tackle 'voice poverty' we pause for an epistemological breath and ponder the implications of power relations in globalising media for storytelling. Various groups with strong oral traditions hold differing, often dichotomous, views towards including Western tools in their society; for instance, indigenous groups have long recognised that electronic recording is a "Faustian bargain" [9] capable of corrupting as well as preserving traditional knowledge. Yet, the success of a vast range of digital storytelling projects often rests on prescribing ways to tell stories [10]. These may emerge from the domestification of recording media in specific social systems and apply the conventions of author autonomy, univocal voice and linearity of the written word.

Our media heritage contributes to complex relationships that shape our beliefs about engagement, participation and dialogue. Consider interplays between the affordances of recording media and views on relationships between speaker and listener, author and reader, game designer and player, filmmaker and viewer. For instance, claims that it is more difficult for a film to evoke empathy than written literature are situated in writing culture and may apply differently to those where storytelling is usually an 'event in time' [7]. Power relations in the ways groups accrue, recreate and divide knowledge and our perspectives of where a story 'comes from' and who is permitted to voice it are also cultural. For instance, views that stories are 'owned' by ancestors or the land are in stark contrast to Western Constructivism, where authors control narrative and listeners determine meaning.
While we are often aware of cultural gulfs in interpreting a story's content or purpose, we can be oblivious to the communicative modes that lie buried in the details of a story's performance, structure and form. Finnegan argues, that our constructs associate with a heritage of writing and these may differ from those in cultures with the oral 'texts' and less mediated storytelling in Africa [7]. For instance, oral narrative in the anaXhosa tradition manipulated time in ways that differ from the devices used in writing. Storytellers created temporal movement within sets of patterns, for instance repeating phrases, segments and motifs, rhythmically across a chronological narrative to engage listeners, invoke emotion (e.g. anticipation) and introduce detail with each repetition [21]. While temporal settings and qualities may distinguish oral performance from the 'timeless spatial text of writing' [7], Xhosa narratives also established spatial relationships. For instance, they linked characters' movements to rites of passage (e.g. the typical journey a woman takes between her houses of birth and marriage) and poignant archetypes (e.g. the sanctuary of home and insecurity of the unfamiliar) [21]. The sensual experience evoked by such patterns may persist in dialogue and while fewer orators perform the classic tales of 'oral literature' [7] today the oral experience "retains its hold on the African imagination" [21].

Conceptions of how we create, represent and interact with stories affect the design of digital storytelling applications. These often reproduce the patterns of other media; for example, one of a few existing systems that supports mobile digital storytelling, Jokela et al's Mobile Multimedia Presentation Editor [11] (MMPE), uses a timeline interface influenced by video- and audio-editing. Thus, the usability of digital storytelling applications often propagates values [19] embedded in other media. Differing views on whether communicative power lies with narrative or visual imagery [1] effect the emphasis of applications but, again, these are cultural. For example, iTell [15], which runs on a computer, is modelled on a process of brain-storming, organizing and writing that is used by experts to teach the "art" of digital storytelling. The 'story-driven' approach behind the interface intends to help users reflect and develop the story (e.g. focus, setting, characters, plot, events) before they cut and edit photos and voice-overs. As most story-driven approaches involve some writing they may inherit constructs which associate a text of words and imagery with "events in space" rather than time [7]. In contrast, habits of visual representing shape 'photo-driven' applications, which lay voice-overs over images. This approach may be comfortable amidst a Western 'hyper-visual' culture, but less at home in cultures that are more aniconic or restrained in graphical representation. Internationalizing interfaces with culturally-sensitive icons or local language makes software accessible to those excluded by textual illiteracy. But, to design applications suited to strong oral traditions, we must enfranchise the voice of the 'other' before we repurpose Western styles of recording or generalise rule-sets by photo- or story-driven approaches [17]. 


\section{Oral Stories in the Dialectic of Design}

Stories may serve as a "dialog between people, cultures, and times" [15] and contribute to the dialectic of design; or the ongoing exchange between the producers and the users of the media. We widely accept the value of storytelling to HCI practice, whether we bring people, or depictions of people, into user-centred design (UCD) or enter users' worlds via participatory design (PD). Voices and bodies telling stories are core to many PD methods [e.g. 13] and the artefacts informing UCD. We gather and engage with data about users and use contexts [20] and manage such data's diversity through stories [6]. We also use storytelling and performance to bring bodily phenomena [14], plurality [8] or egalitarian efficacy to envisioning design. But, the tools we use to involve users in design and gather, depict and engage with their lives or interactions are riddled with particular traditions of storytelling and media use. For instance, diary-studies, photo-logs, scenarios and design documentaries are culturally-situated communications [8], and even sketching in rapid prototyping relies on habits of graphical representation. We do not intend to devalue how storytelling engages designers analytically with accounts about users or empathetically with users' felt experience [20], but contest that activities emerging from the ways of doing and saying of Western, mediated orality may obscure nuances that constitute storytelling 'elsewhere' [18]. Our own 'authoritative' voices can silence the 'other' or relegate their voice into the background of the dialectic of design.

Our use of face-to-face storytelling within HCI is located [18] and contextualised in an extensive ecology of written or digitally mediated interactions. This contrasts with the contexts of remote people, who survive by co-operating, exchanging knowledge and attending to interpersonal relations face-to-face, and of those who emphasise communicating, with each other and with their environment and history, in speech, song, music, drama or crafts. Everyday acts of communication in places with low population densities or few modern amenities differ from those in urban places where structures are more extensively shaped by media. Thus, regardless of their textual literacy rural inhabitants often encounter disjuncture between technology and their lived experience that reflects incongruence between rural life and models of communication produced in urban places [3]. For instance, video conferencing and social networking applications match less well to the interactions of farmers or other rural residents than to the interactions of office workers or urban inhabitants [3]. Thus, to design for rural contexts we must hear the voice of rural people in their everyday acts of saying and listening.

\section{OUR DIALOGIC IN PROTOTYPING AND ENTHOGRAPHY}

The design activities that ultimately resulted in our current prototype originated through the confluence of formative development of initial prototypes and in-depth situated data gathered in a remote, rural community in the Eastern Cape of South Africa. The initial prototypes were tangible artefacts around which we could conjecture how use might diverge from ways of doing and saying depicted ethnographically. Further, lab-based testing and some field use of those initial prototypes, coupled with ethnography, helped us to conceive methods to involve rural participants in design. To explain this we summarise how we developed our initial prototypes and, simultaneously and independently, gathered data on rural communication. Then we consolidate the outcomes of interrogating the initial prototype and the ways locally enacted practice contributed to developing methods to further inform design.

\section{The Initial Mobile Digital Story Prototype}

To explore possibilities for an easy-to-use system to allow people to record their stories on mobile platforms we drew on interaction scenarios apparent in our personal experience of storytelling and digital story-telling projects globally [17]. We based the first seven preliminary prototypes on a usage scenario in which a user combines a set of three photos with an audio record of reading from a scripted story. In the first design iteration we created low-fidelity paper prototypes of two different approaches. In the storydriven approach users record a narrative first and then add in photos; while, in the photo-driven approach users add photos to a storyline and then annotate these by recording audio. We tested the paper prototypes against the usage scenario and incorporated insights of a heuristic evaluation into a second design iteration. We again developed prototypes of story-driven and photo-driven approaches in our second iteration which we evaluated, using PowerPoint with six university students. Based on evaluating the second iteration's interface elements (e.g. button placement) we created high-fidelity Flash Lite prototypes running on a cellphone. We evaluated the third iteration with eight students and, based on their preference in the context of the usage scenario, we used the photo-driven approach to create an interactive prototype. We tested this last Flash Lite prototype against the MMPE [11] by evaluating how efficiently ten students added photos to a storyline and recorded a pre-scripted story [17].

Few people in our evaluations had heard of digital storytelling but almost all suggested a usage scenario for our system; from "telling a friend about the club I'm currently at" to "using it with people in the AIDS clinic I volunteer at". These scenarios imply an 'on-the-move' spontaneity but may differ from the verbal presentations of closely-knit, rural communities with strong oral literature traditions, such as in African communities [7] like the anaXhosa [21]. While $80 \%$ of our prototype evaluators were African, they were all city-based, university students who are accustomed to Western media. Thus, to explore a more elaborate interaction scenario, where users construct their own story, rather than read a script, we also developed another prototype in Mobile Python. This prototype was informed by usability outcomes from the story-driven prototype in our third iteration but enabled users to record and select their own audio and photos. We (authors 1 and 4) tested it in-situ in a minimal way in the Eastern Cape with Sibongile, a man who was known locally as a great 
storyteller, and by creating our own digital story. The difference in these evaluations alerted us to the prototypes' materialisation of assumptions about storytelling that are embedded in mediated orality, writing and 'hyper-visual' culture. We (the two non-African researchers) preferred to develop a storyline over time in situ and were more photodriven than Sibongile who mentally composed his story and included only two photos which he said had a limited relation to his story about a trip to a city.

\section{Ways Of Doing \& Saying in Eastern Cape, South Africa}

Our ethnographic perspective on storytelling is informed by data gathered independently of developing initial prototypes and is situated in Lower Ndungunyeni in the Wild Coast of South Africa's Eastern Cape. Isolated by topology, neglected by successive regimes and with a history of resistance to colonists, famine and invasion means that everyday life anchors to customary communication and power structures and traditional habitation and land-use. Most residents can trace their ancestry to the settlement of the $50 \mathrm{~km}^{2}$ area at least eight generations ago by the Khonjwayo, one of six Chiefdoms descending from a distinct tribal monarchy. Families live in umzi, which are informally distributed clusters of thatched, mud-brick rondavels, fronted by a garden for subsistence crops and connected by paths across hilly common grazing land. Formal, legislative institutions are separate from custom and daily practice; for instance people elect politicians but are closer to Headmen who inherit leadership patrilineally. Ndungunyeni's 20,000 inhabitants are acutely impoverished and, with remittances, pensions and child benefit, $80 \%$ of families survive on less than $10 \%$ of the national, median income for a working white man. Even those benefiting from a relative's temporary migration to a city or able to diversify their income have limited local access to 'modern' facilities [3]. There is poor transport, no sanitation and most of Ndungunyeni has no grid electricity, although clinics, some schools and a few homes have solar power.

Insights on storytelling, oral and digital communication emerged over 18 months as we formed relationships, interpreted priorities, discovered design opportunities in the ad-hoc details of daily life, and undertook socio-technical experiments [3]. We have described these as four phases which began by establishing relationships, via emails and a short visit, with a volunteer in Transcape, a Non-profit Organisation (NPO) and with the son of Ndungunyeni's senior Headman. This enabled us (author 1) to live in the village of Lwandile and focused our attention on interfaces between traditional leaders, the community and local and remote agencies on development projects. In Phases 2 and 3 we lived, according to local norms, for two months to collect data on domestic and community life and participate in activities. Phase 4 began when we left Lwandile and included two further visits, separated by 9 months encompassing 18 days in situ. In situ we recorded data using handwritten notes, 650 photographs, of which villagers took 200, and 12 hours of video. Ex-situ we integrated logs of email, SMS, phone calls and Facebook interactions, verbatim and video transcripts, 'thick' descriptions, and derived themes hermeneutically.

\section{The Context of Our Relationships to Everyday Storytelling}

Storytelling and orality is contextual and the meanings we construct about local storytelling emerged in relation to diverse experience in Lwandile. Thus, here we summarise the settings and activities in which we observed the ways local people verbalise action, tell stories and entextualise their values and meanings in those speech acts. To begin with we gained insight by passively observing visitors to the umzi in connection with the Headman's duties (e.g. resolving disputes, signing hunting permits) and during impromptu contextual interviews, about everyday life and priorities, with the Headman's family. Daily interactions developed swiftly with the elder sons and with women during domestic duties. With increased familiarity we conversed about local issues with local teens, villagers and others in the umzi or in the village, and gained insights into the Headman's senior son's priorities for development and pursuing the Khonjwayo Chieftenship. As time went on we talked with the Headman's son-in-law, a Chief in another area, and local people in Coffee Bay (a tourist area an hour away) and in Transcape's premises where villagers mix with Afrikaan and foreign volunteers. Our discussions widely varied in content and purpose. We noted villagers' interactions with each other as they travelled on local transport, between villages and en route to the city (Mthatha). We observed village meetings; meetings that led to founding a new independent NPO; and, a tribal meeting at the Palace where we were formally introduced to the Queen. We also accompanied the Headman's son on trips to Transcape NPO and to look for records at Municipal Archives and the Palace. We also gathered data on formal performances, for instance at a school's official opening by provincial dignitaries and in events that arose because we were there. For example, we introduced the new NPO to The National Archives \& Records Services which led to hosting a 3.5-day workshop on Archives in Lwandile School, attended by over 50 villagers. The workshop was based on long oral presentations by the departments of Land Affairs and Environment; the House of Traditional Leaders; the Chief's emissary; and, the local Councillor. We observed media use locally and computer use at an Internet café in Mthatha, in a Coffee Bay tourist hostel and in Transcape's Education Centre. We discussed villagers' media preferences and use in generating income and gathered data on phone handset models from the start of airtime sales in a spaza (local shop).

\section{'Experiments' in Digital Communication \& Story-telling}

Our situated technology experiments were originally motivated by the Headsman's senior son's interest in using technology to attract attention to, and funds for, his local upliftment initiatives. In Phase 2 we introduced him to various Internet facilities to search for information, and view NGO and Facebook web-sites and from Phase 3 spent an hour a day teaching his sister to set-up solar power, send 
emails and explore the Internet for information of interest to her. We also illustrated the Internet to children and sent emails for villagers who visited on errands, to charge cellphones or through curiosity. In Phase 4 we extended the Wi-Fi network, set up email and accounts and a group on Facebook, which yielded data on interactions around photos and text remotely. Various digital storytelling activities arose in situ. Some were intentional, for example we created a blog to which the Headman's son and daughter uploaded photos and typed short texts. Other digital storytelling was impromptu. The Headman's son used his camera phone in discussions with dignitaries at the opening of a new school; family members orally described their photos or video, such as those they took at a party to honour the Queen; and, we videoed interviews with the Headman and while walking with family members around Lwandile.

\section{Provoking A Probe}

Insights into local priorities, communication practice and technology-access in Ndungunyeni confirm the potential value of a mobile digital storytelling application. Up to half of 9-year-olds in Lwandile cannot read, partly because school children are taught literacy in English but villagers usually speak isiXhosa. Illiteracy is not stigmatized; rather, social practice and preferences for media, when present, emphasize orality, song and dance. Villagers have limited access to TV and their main media are radio and cellphones. They make calls more rarely and abruptly than they would like, as airtime is prohibitively expensive, and use SMS as it is cheaper. Villagers were enthusiastic (e.g. in the Archives Workshop) about recording local stories and felt video might preserve their heritage in ways writing cannot. But, they also noted that recording must be compatible with the features of orality and performance that construct their local identity and not threaten social structures in the way that elders attribute to American movies. This provoked us to query our initial concept through an ethnographic lens, and structure new activities to hear users' voices in design.

\section{Supporting Agility \& Serendipity}

By inferring the ways the initial prototype might have been used in the storytelling situations we observed in Lwandile we realised that a story-driven approach may not serve a storyline that emerges serendipitously. Villagers' accounts were often prompted by cues in the landscape (e.g. the tree that a villager's brother planted); in ancestry (e.g. the Headman's lineage); or by images (e.g. a sequence of photos taken at the King's party). In our blogging activities villagers often found it difficult to think of a story without such resources. So we sought a loose, non-prescriptive way to enable participants to create storylines by drawing on the representational, physical or social. The mechanism to do so needed to take into account, firstly, that villagers are unfamiliar with the mutability of software development as most, with the exception of our experiments, have never used a computer or feature phone. Secondly, villagers treat writing as special and sketching and writing materials are not available locally [2] so paper prototyping, typically used to defy rigidity and determinism is unsuitable. To give participants a flexible and easily observable way to record and combine photos and audio we decided to use a pair of low-end camera-phones and their rudimentary default image and voice recording software. We dedicated one phone, Nokia 6600, as a camera and the other, Nokia 6630, to record audio. This technology probe had enough ambiguity to reduce constraining use but aligned with villagers' experience as most, over 15 years, own or share a basic phone (e.g. Nokia 1100).

\section{Probing Collaboration in Storytelling}

Our initial conceptualisation of mobile storytelling as an individual activity is discordant with villagers' proximity, shared use of phones and communication norms. They devote significant time exchanging views in meetings and these protocols of speaking and listening contribute to cohesion, shared identity and security. We thought that a workshop in which participants used a camera-audio phone pair in groups would enable us to observe task division and requirements for collaborative elements. We were eager to notice diversity in collaborating in audio recording as we have observed gender differences in patterns of turn-taking that manage spoken interaction and participation. In male and mixed groups people listen quietly until a speaker finishes but, in female-only groups, women often repeat items in synchrony with each other [2].

\section{Enabling Core Values to Localize Useability}

Our experiences in Lwandile led us to question the values underlying the usability of our prototypes. For instance, we evaluated our initial concept on efficiency criteria but villagers prioritise launching and maintaining relationships over speed in face-to-face dialogue. Consider the way the Headman's son first ingratiated the Education Minister, using photos on his cell-phone, before illustrating Lwandile School's need for resources; and the prolonged, debate in village meetings that feeds into a Headman's decisionmaking about collectively-owned resources. Realising that speed is less salient in dialogue than consensus or 'friendship made by speaking' prompted us to reconsider values affecting expressivity and useability. Villagers in Lwandile emphasize displaying unity in everyday life, such as expressing solidarity and belonging by joining in songs each day. They do not recognise such inter-dependence as a trait defining Western constructs of personhood [2]. Further, while some African traditions perform tales to big audiences, Xhosa story-telling was 'essentially a private matter' carried out amongst those who knew each other well to ensure rapport [7]. Thus we spread activities over consecutive days so participants could involve others outside the workshop in their own way and used part of the phone-pair probe to record data on interactions remotely.

\section{Providing Privacy in Participation}

We sought to reduce the effect of inevitable power relations on use of our phone-pair probe. For instance participants might have felt shy about recording opinions for us to scrutinize. We sought to respect the boundaries that enabled people in Lwandile to separate their intimate locale from 
external structures and outsiders. One such boundary is language, so we decided to ask participants to record stories in isiXhosa even if they knew English.

\section{Probing the Materials Used to Convey Meaning}

Concepts about people's use of resources to prompt and progress storylines and convey meaning in stories are embedded in the story- and photo- driven approaches of our initial prototypes. However, we noticed that the landscape progressed the narrative in storytelling in Lwandile and prompted recollections. For instance, the Headman gestured across hills in reminiscing sending a messenger on a horse and his son animated stories of his youth by indicating a forest. By encouraging participants to take as many photos as they wanted between two workshop sessions we hoped to discover relationships between content in photos before their integration into stories. Thus, even without necessarily understanding the audio, sets of photos might provide insight into both the experiences that prompted participants' stories and storylines and they choices they made in integrating and balancing photos and audio.

\section{Probing Converging Perspectives in Narrative Structure}

Our initial prototypes instantiate rules about unidirectional story and timeline but more recent trends, such as online story mash-ups, support multiple viewpoints around a theme. While villagers' individual narratives certainly seemed to have a story arc resonant of a singular linear flow, group communication arose through orthogonal relationships between diverse perspectives. Importantly, villagers seemed to pursue unanimity through disparate tangents so their voices seemed interdependent. This may be a consequence of oral narrative's inherent malleability, to history and politics, and a need to unify community and maintain elder and patriarchal authority. In all group communications involving dissimilar views and ideologies villagers emphasised that resolution emerges by listening to multiple perspectives not by overt coercion. For instance, neither the Headman or the incumbent Chief's emissary expressed disagreement in re-telling their genealogy; they simply told the same story of their lineage which differed in one fine, but critical, detail; who was the firstborn of twins some eight generations ago. Thus, we sought to sensitize ourselves to participants' management of interactions around multiple views; for instance, if they collaborated did they favour a unitary narrative, interrupt linearity or connect various directions from disparate parts?

\section{Generating Empathy Between Designer \& User}

As we reflected we encountered difficulties in uniting our initial storytelling concepts with our insights on villagers' storytelling and role-playing these insights using the early prototype. We were anxious because we knew Lwandile villagers felt outsiders did not articulate the meanings that entwine their identity with a setting in which their kin have resided for generations. We observed how the features of, and material used in, their storytelling join to expectations bound to community, place and being Khonjwayo. Lwandile's isolation and a daily-life spent outdoors means villagers are not anonymous and from birth to burial, and beyond, their identity is etched into the land and their stories index to the furniture of rural life. Relationships are encoded, symbolically and syntactically, in the landscape; customs define where a villager can establish an umzi and as they are buried in their umzi ancestors' graves are nearby. Name sounds acutely associate with umzis, as isiXhosa language carries in the open-air, and names carry stories. Thus, we sought to ensure that our activities would sensitize us to facets of participants' identity. We hoped that the 'returns' from our phone-pair probe, such as ambient or contextual content of photos or audio and the resulting digital stories, would engage ex-situ designers (e.g. authors 2 and 3) empathetically. We also hoped that video of the workshop would enable us to link our more ephemeral experience of participants' worlds, through the probe returns, to concrete interactions with technology.

\section{DEPLOYING THE PROBE}

We deployed our phone-pair probe in the village of Tschani, $15 \mathrm{Km}$ from Lwandile. This enabled us to host the accompanying design workshop in Transcape's Education Centre nearby; which, in normal circumstances, has access to electricity. We ran the workshop on two consecutive afternoons and recruited six participants via the NPO, five of whom lived in Tschani. Participants included two young men: Bafundi (20 years) and Sphiwo (22 years), who occasionally attend the Centre; and four women, two preschool teachers at the Centre: Kholiswa (23 years) and Nolutho (33 years) and two of their friends Celine (22 years) and Noileka (23 years). Three participants were fluent in English and the others understood a little but would not speak in English during the workshop; so one participant, Nolutho, translated our explanations. At the end of both sessions we compensated participants with dinner. We used a mini solar panel to charge the phones due to a power-cut during the workshop and had to substitute a Nokia N95 for one Nokia 6630 phone to record audio.

\section{Workshop Session 1: Overview \& Learning to Record}

At the start of the first afternoon, as participants arrived and looked at the phones on the table at which we sat, we discussed cell-phones. Bafundi, Noileka, Kholiswa and Nolutho own Nokia 1100s, Sphiwo a low-end Samsung, and Celine a Nokia 2300. They were all intrigued and enthusiastic about the N95 and asked us the cost of such a model. Then we outlined that the workshop's purpose was to inform designing functionality for digital stories and participants' roles as 'user-researchers'. We simplified some explanations, such as that we were designing a phone, rather than software, that would be affordable locally. Then we demonstrated, on a laptop, a digital story that we had created a day before. The story was a comical parody of author 1's experience in Lwandile as she learnt to carry a bucket of water on her head but was set in Tschani with photos of villagers undertaking their ordinary activities. As we had hoped, participants found the story amusing and accessible; but we also emphasized that digital stories can be more serious, historical, or informational. 
Before using the phones we discussed participants' views on what they might use digital stories for (e.g. education, training, news to family, fundraising). But, participants were reserved and the men seemed distracted, relying on the women who worked at the Centre to engage. We realised that although we modelled our introduction on the didactic delivery we observed in the Archives Workshop at Lwandile School, this might not match our participant's expectations. The Archives Workshop was organised by villagers and involved, mostly, male presenters. In contrast, our digital stories workshop was facilitated by white women (authors 1 and 4) in an Education Centre, which adopts a more constructivist approach than the formal and informal education in local schools or village practice.

Participants became more animated as soon as we began activities with phones. We explained that they should use the phones over the next 23 hours to take as many photos as they wanted (of which they would receive printed copies) and incorporate into stories in any way they preferred. They formed three groups according to friendship and home location: the two young men, Bafundi and Sphiwo together; Kholiswa with Celine and Noileka; and, Nolutho on her own as she lives in a distant village. We demonstrated and assisted use of the Nokia 6600's Camera and Gallery software and then participants practiced in the NPO's grounds. We observed them take photos in their groups and show their photos to the subjects in them (e.g. workers and visitors) and to other participants. Then back in the Centre we reviewed deleting photos and, while recharging the 6600s, demonstrated the 6630's and N95's Voice Recorder software. All participants eagerly engaged with audio and unself-consciously recorded their voices. Before ending the first session we reflected on storytelling to emphasise there is no right or wrong way to tell a digital story. The women had ideas about stories but the men felt uncertain asked for guidance on composing storylines. We agreed that it can be difficult to think of a story and encouraged their confidence by facilitating a discussion of stories, such as recounting the events of a recent village football match.

\section{Workshop Session 2: Creating \& Assembling Stories}

While participants arrived on the second afternoon we chatted about the photos they had taken since the previous afternoon and problems they encountered. Nolutho took 60 photos on her own; Bafundi and Sphiwo took 41 photos together; and, the other group took 78 photos, which were mostly taken by Kholiswa as the battery was flat by the time Celine had the camera-phone. Participants noted their disappointment in being unable to take photos in the low illumination of their homes, which have few windows and no electricity, so $30 \%$ of Kholiswa's photos were black.

After we had recapped on workshop aims we reviewed making audio recordings and asked participants to reflect upon their stories. We asked whether participants thought it would be easier to: record speech and then find photos to fit, think of a story then decide on suitable photos and record speech; or view photos and record speech. In their

\begin{tabular}{|c|c|c|c|c|c|c|c|}
\hline \multicolumn{8}{|c|}{ Content of Photos \% of Total Taken by Group } \\
\hline \multirow{2}{*}{\multicolumn{2}{|c|}{ Main subject of Photo }} & \multicolumn{6}{|c|}{ Group } \\
\hline & & KCC & & $\mathbf{N}$ & \multicolumn{3}{|c|}{ B\&S } \\
\hline \multicolumn{2}{|l|}{$\begin{array}{l}\text { People } \\
\text { (of whom are children) }\end{array}$} & $\begin{array}{c}87 \\
(36)\end{array}$ & & $\begin{array}{c}68 \\
(17)\end{array}$ & \multicolumn{3}{|c|}{$\begin{array}{c}80 \\
(22)\end{array}$} \\
\hline \multicolumn{2}{|l|}{ Buildings or interiors } & 6 & & 10 & \multicolumn{3}{|c|}{0} \\
\hline \multicolumn{2}{|l|}{ Livestock } & 8 & & 7 & \multicolumn{3}{|c|}{15} \\
\hline \multicolumn{2}{|c|}{ Landscape/garden/grass } & 0 & & 15 & \multicolumn{3}{|c|}{5} \\
\hline \multicolumn{8}{|c|}{ Audio to Photo Ratio in Stories } \\
\hline \multicolumn{2}{|c|}{ 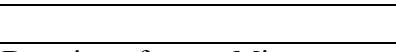 } & KCC & $\mathbf{N}$ & \multicolumn{4}{|c|}{ BS } \\
\hline \multicolumn{2}{|l|}{ Duration of story. Mins } & 3.18 & 1 & 1 & 1 & & 1 \\
\hline \multicolumn{2}{|l|}{ Number of photos } & 22 & 7 & 6 & 6 & 3 & 5 \\
\hline \multirow{2}{*}{$\begin{array}{l}\text { Duration of voice-overs } \\
\text { per photo. secs }\end{array}$} & $\min$ & 3 & 8 & 5 & 5 & 17 & 5 \\
\hline & $\max$ & 25 & 11 & 20 & 12 & 24 & 11 \\
\hline
\end{tabular}

Table 1. Summary of photo contents and story properties

discussion they were undecided between these methods. Participants then separated into their groups, discussed their stories and recorded audio for 90 minutes. Groups made varying number of stories (Table 1) and some did not finish all of the stories they intended. Bafundi and Sphiwo deleted the sound-clip to one story and Kholiswa's group had a set of photos they took to use in a story about her father counting sheep in the morning. To conclude the session, and enable us to assemble stories after the workshop, each group went through their audio, photo and stories. While groups constructed stories in distinct ways they all tended to co-ordinate voice-overs in one audio clip with a sequence of photos. We had mentioned that one option was to associate a photo with a short audio clip but they preferred to record voice-overs of a minute, with the 6630 , or longer with the N95; and use the pause function. Thus, participants listened to an audio clip and indicated to us the time that it should synchronize with a specific photo. This was troublefree and we easily cut stories together after the workshop. A week later we sent albums of photos to participants and DVDs for groups to view their stories at the Centre.

\section{Probe Returns}

The content of participants' photos (Table 1) included a range of details about everyday life and values, some candid and intimate; from pigs, to puddles to a naked infant peeing. There were stunning photos of landscape, of sunlight through branches or haloing a cow. Most contained people, in homes, gardens or fields, often undertaking activities (e.g. cleaning, cooking). Participants' stories also focused on people and, for the women, these were biographical. People were in all but one of the 22 photos in a group's story about Kholiswa's infant daughter's routine from awakening to walking to school. Nolutho featured in all photos of her story about gardening, showing that she enlisted a friend. Bufundi's and Sphiwo's stories were staged performances with props. Two seemed deliberately comical: chasing pigs from a home and an infant using a cell-phone; but two seemed to be a gentle satire about their life, they alluded to issues of alcoholism and producing sufficient melons to feed a huge family. 


\section{THE SECOND PROTOTYPE}

We (authors 1 and 4) noted many ideas in the workshop as we observed participants collaborating on audio or linking audio with photos in different ways. We uncovered other design requirements when we (author 1) assembled digital stories and participants' photo albums and steered the designer-developer (author 2) through photos, stories and notes and video of the workshop. We perpetuated, added and disputed design ideas, to develop our most recent prototype, by reviewing the video of participant's interactions in the workshop. We watched the video eight times in total for inspiration and analysis, but rarely in entirety. Rather, we would watch an hour or so, pause to play with and sketch an idea and then query the idea by reviewing the video. The first two viewings familiarised the designer-developer (author 2) with participants and inspired some design requirements. This familiarity enabled us (author 2) to gather less palpable ideas in subsequent viewings, such as the interface's general feel and ways to combine all design requirements. The video alerted us to subtle interactions; for example, we conceptually fused the two phones when Nolutho held them closely together in recording her story; and, we noticed rhythms as Kholiswa's group took turns in speaking. We posed numerous scenarios to explore and refine the design space and, iteratively, improve ideas. For instance, we rejected an initial idea of a script writing tool, based on Bafundi's and Sphiwo's use of a handwritten storyline, because Nolutho and Kholiswa's group built or adapted a storylines in more situated way. Here we outline the provenance of design features, compare the current prototype with similar software and reflect on its role in addressing relations in our ongoing dialogic.

\section{Design Features \& Their Provenance}

Our current prototype runs on Symbian S60, the most prevalent operating system for feature phones globally. Participants' mutual physical proximity in the workshop confirmed earlier observations that viewing the cell-phone as a 'personal device', affording use by one person at a time, is based upon Western habits of 'personal space'. Thus while we designed the prototype for mostly single user scenarios often features reflect participants' collaboration. For instance, we aimed to create a flowing interaction inspired by the way women in Kholiswa's group took turns to say parts of the story, associated with each photo, and fluidly and intuitively knew when to speak. Thus we synthesized interaction ideas and requirements into an interface that might respond to the storyteller as a friend might; much like the way Sphiwo located photos on one phone to help Bufundi as he recorded audio on the other.

Once open, the prototype presents the user with a centrally positioned tool bar of icons for adding, selecting and rearranging photos and recording audio (Figure 2). This reflects participants ease in using the vertical icon toolbar interface of the voice recorder application but difficulties using text-based menu systems in the probe. We designed for flexibility so that the user can begin by recording audio or adding photo/s because the three groups in the workshop had different story recording strategies. If the user clicks the 'add photo' icon the application launches the default image gallery to enable selecting from thumbnails and filenames. We based this decision on observing participants use of thumbnails in the phone's gallery application.

When the user has selected all the photos s/he requires, at that time, the prototype displays them in a storyboard carousel of up to 11 photo thumbnails in increasing sizes, scaled to make best use of screen real estate (Figure 2). This arrangement aims to reduce the time overhead that participants encountered in navigating through photos in a linear system and memory load in recalling a long sequence of photos. The carousel also enables easy navigation and may assist users in planning a storyline; for instance, Bufundi and Sphiwo had written storylines on paper which they consulted to help them co-ordinate audio with photos. Sometimes in between recording audio they annotated their lists suggesting that during the process of recording they realised a more effective order to convey their story. Thus the carousel offers users a way to envisage alternative story structures, such as possibilities for patterns and repetition, as photos do not appear along a vertical or horizontal.

The user can add photos and change the order of photos on the carousel at any point before recording audio. This is

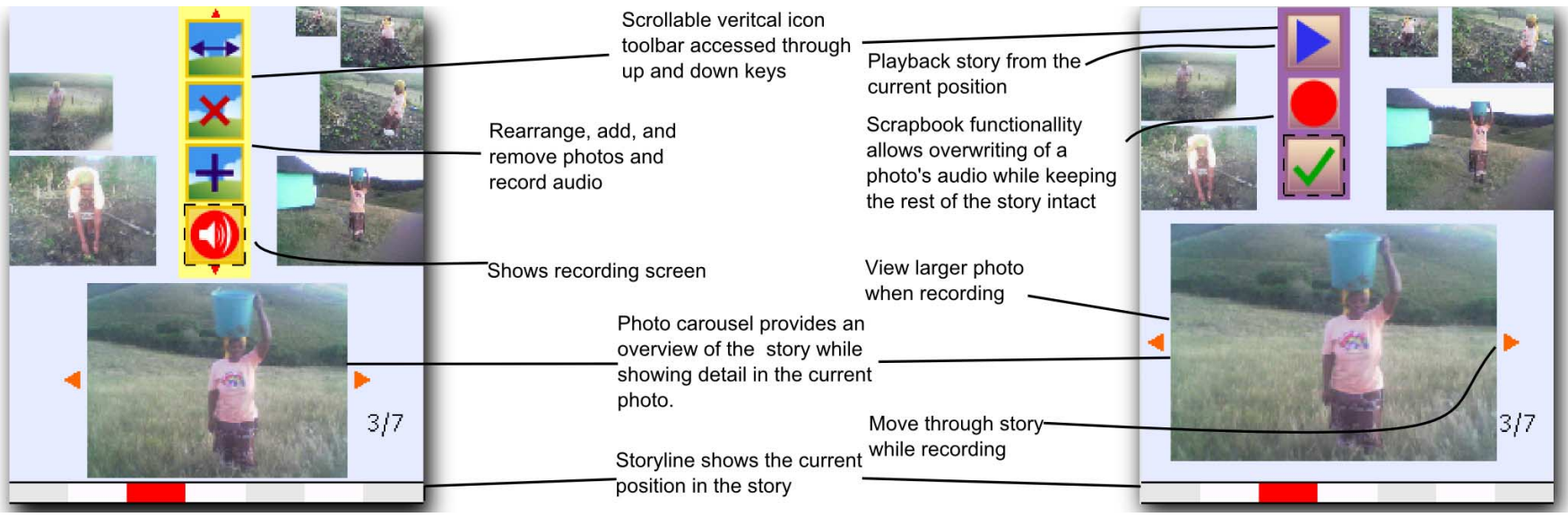

Figure 2. Mobile Digital Storytelling prototype and elements of the Storyboard (left) and Recording (right) interface 
vital as Nolutho, Bufundi and Sphiwo wanted to alter the order of photos during or at the end of recording an accompanying audio. We used animation so the photos move around the carousel when re-ordered to help reduce errors, such as Nolutho's confusion about the direction of her photo sequence. The user can include multiple copies of photo in a story which may serve in revisiting a feature or the emphasis and rhythm that similar photos provided to Kholiswa's group's story-telling. The user can also take photos from within the prototype by launching the camera.

We intend the recording photo carousel of our prototype to also flexibly enable users to draw upon visual cues in telling their story. Most participants held the two phones next to each other while recording audio, drawing on photos as memory prompts. To support this, the prototype enables users to view photos in the carousel. They can record the audio on a photo-by-photo basis; as observed for two groups who paused recording after viewing each photo and resumed as the next photo was displayed. Alternatively, users can view the next photo of the story while recording audio. One group consulted written storylines on paper to determine the next photo against which to record audio. As Bufundi recorded audio Sphiwo located the next photo in the sequence to help him. The carousel permits the user to move to the next photo without having to pause recording, and thus, enables users to record their own rhythms in speaking. Throughout this process the prototype captures all interactions with the carousel for the user to draw upon in photo transition timings during playback, for instance to map photo timings to vocal patterns.

The prototype allows the user to record audio in one go or record and playback in segments. Recording the story in enterity might suit users like Sibongile, the expert storyteller who used our initial prototype, or people with scripts. The user can playback a recorded audio segment; just as Bufundi and Sphiwo replayed an audio segment they had just recorded, to check it sounded right. The prototype also enables users to supplement audio because when Nolutho listened to her audio after re-ordering her photo sequence she said 'I need to explain more' and created another sound clip to insert into the middle of her story. Recording in segments also offers the capacity to tag photos in a serendipitous manner and collect a 'scrapbook' of audio-tagged photos. Thus a user can construct a story in pieces and iteratively refine segments until a final story emerges; which might support those who compose by collage and workshop participants who situated stories in a journey or had difficulty in formulating a story idea. It also supports shared storytelling as multiple users may use a phone to contribute their own story segments.

\section{Contrasting the Prototype with Similar Software}

Our current prototype differs from the details published about other mobile digital story applications. Firstly, unlike either Jokela et al's MMPE [11] or Jones et al's StoryBank [12] our prototype allows flexible usage of audio and/or photos. MMPE and StoryBank were modelled on another media (e.g. PowerPoint) or designed to suit the story-format of the Digital Storytelling movement $[5,10]$ and have a task flow for integrating audio and photos. Secondly, like StoryBank, our prototype avoids written text and presents icon-based interfaces to the user. In contrast, MMPE uses text menus and permits users to include text and stickers in their presentations. Thirdly, unlike the other software our prototype allows users to iteratively record the story's narrative and craft the audio experience, with or without photos. Finally, while StoryBank and MMPE allow for one recording of 1-2 minutes our prototype does not restrict audio duration or quantity of photos. The unique affordances for audio seem vital for rural African users.

\section{Re-plying a Dialogical Approach: A Further Story Probe}

Our current mobile digital storytelling prototype is an unfinalized understanding of our user needs; but not only because we are still evaluating it. The prototype emerged from participants and our active construction of stories, storytelling and digital storytelling situated in the phonepair probe and the workshop setting. Our experience in this construction alerts us to power relations in the workshop that might affect whether participants felt that they were "experts at their stories" [5] and their use of the probe. Here we unmask our prejudice in the workshop and considering its returns, as part of the process of understanding others through understandings of ourselves.

Various factors symbolised us as 'authorities' on digital stories: the technology we brought; the workshop's separation from ordinary village life; and, values associated with Western education and the NPO. Sensitivity to specific 'acceptable' ways to tell digital stories may be conflated by existing perceptions of storytelling. For instance, in village life some people, like Sibongile, are esteemed as storytellers, perhaps reproducing the high regard for traditional orators [7]. So, we may not have presented our probe with the ambiguity we intended. We may have affected Bufundi's and Sphiwo's storytelling since, after an initial lack of confidence, they set up photos and used humour in a similar way as the story we displayed. Other power relations might exist between Kholiswa and her group members. Like Nolutho, Kholiswa tells stories to infants at the pre-school and the Centre's manager had spent 18 months training her tell stories. Despite such influences we also noticed qualities in participants' vocal rhythms and patterns that seem aligned with those distinguishing the African oral arts [7] and daily speech [2].

Content in photos might determine subtle aspects of story structure. For instance, some participants' stories, like the one we displayed, were about people doing things and thus had procedural aspects. We taught participants to take photos first because it was clearer to initiate participation across the language differences using visual cues and we sought give participants plenty of time to think of stories, take photos and record audio. However, learning to use the camera first did not prevent participants from determining their stories before they took photos. Further, stories often 
have sequenced locations that echo the detailed spatial movement we see in traditional Xhosa oral tales [21].

Unmasking bias is vital to the quality of HCI4D's engagement with communities and to avoid the risk of 'development tourism' [4]. We simply cannot know users if we do not learn about ourselves in relation to them, our activities and settings. This never ends regardless of time spent in situ. Pragmatically any design process that introduces concepts across cultures will always be peppered with dilemmas in participation. This is a power of ambiguous deployments and how we now use our current prototype to gather data. Participants in our more recent deployments in Eastern Cape and rural Kenya and Namibia, individually or with others, audio-tag photos and tell stories and story segments by audio or photo. Thus, the prototype continues to enrich our dialogue with users, stories and their settings, showing us differences between storytelling in different places and expressing participants' ideas for using digital stories in their own widely varying initiatives.

\section{CONCLUSION}

We set out to design a mobile digital storytelling application but instead we refined a culturally informed technology probe to gather data in storytelling. We chose not to test, and then refine, our initial prototype in a rural community as our ethnography revealed our initial concept was profoundly localised in Western storytelling. Thus, we devised a method to explore digital storytelling in a more nuanced way. This experience sensitized us to our relations with stories in engaging with users and inspires us to challenge others pursuing HCI4D to explore, dialogically, how our relations with concepts in methods arbitrate how we align understandings about ourselves with users. Our prototype is a digital voice that speaks to $u s$ about allowing users to express themselves in design. We believe such a voice will be a key tool in diverse future projects.

\section{ACKNOWLEDGMENTS}

We are deeply grateful to Lwandile's Headman and villagers, Transcape (www.transcape.org), Tschani workshop participants and Bill Tucker, (University of Western Cape) for enabling these links. We thank Nokia Research, Kenya for donating handsets and the Telkom Centre of Excellence for partial funding.

\section{REFERENCES}

1. Balabanovic, M, Chu, L L \& Wolff G J (2000) Storytelling with digital photographs CHI 00 564-571.

2. Bidwell, N J (2009) Anchoring Design to Rural Ways of Doing and Saying. INTERACT09. IFIP \& SpringerVerlag: Lecture Notes in Comp. Sci. 686 - 699

3. Bidwell, N J \& Browning, D (2009) Pursuing Genius Loci: Interaction Design And Natural Places. Pers. Ubiq. Comp. 217

4. Chambers, R. (1994). The Origin and Practice of participatory rural appraisal. World Development (22) 7
5. Crook, M (2009) Radio Storytelling and Beyond. In Story Circle: Digital Storytelling around the World. Hartley, J. \& McWilliam, K. eds. Wiley-Blackwell

6. Erickson, T (1996) Design as storytelling, Interactions 3 (4) $30-35$

7. Finnegan, $\mathrm{R}$ (2007) The oral and beyond: doing things with words in Africa. Oxf./Chicago: James Currey/ UCP

8. Gaver, W (2007) Cultural commentators: Non-native interpretations as resources for polyphonic assessment Int. J. Human-Computer Studies 65(4) 292-305

9. Ginsburg, F (1996) Mediating culture: Indigenous media, ethnographic film, and the production of identity. In: Fields of Vision, L Deveraux, R Hillman Eds. Univ of Calif. Pr.

10. Hartley, J \& McWilliam, K (2009) Computational Power Meets Human Contact. In Story Circle: Digital Storytelling around the World. Hartley, J. \& McWilliam, K. eds. Wiley-Blackwell

11. Jokela, T, Lehikoinen, J T \& Korhonen, H (2008) Mobile multimedia presentation editor: enabling creation of audio-visual stories on mobile devices. $\mathrm{CHI}$ 08 63-72

12. Jones, M., Thom, E., Bainbridge, D., and Frohlich, D. (2009) Mobility, Digital Libraries and a Rural Indian Village. Proc. Joint Int. Conf. on Digital libraries. JCDL '09, ACM Pr. 309 - 312

13. Kensing, F \& Muni-Madsen, A (1993) PD: Structure in the toolbox. Comms of the ACM 36(6) 78-85

14. Klemmer, S, Hartmann, B, Takayam, L (2006) How bodies matter: five themes for interaction design. DIS 06 140-149

15. Landry, B M. \& Guzdial, M (2006) Learning from Human Support: Informing the Design of Personal Digital Story-Authoring Tools. J. of the Int. Digital Media \& Arts Assoc., 3(1) 106-119

16. Ong, W J (1982) Orality and literacy: The technologizing of the word. London: Routledge.

17. Reitmaier T \& Marsden G (2009) Bringing Digital Storytelling to the Mobile. INTERACT09. IFIP \& Springer-Verlag: Lecture Notes in Comp. Sci 750-753

18. Suchman, L (2003) Located Accountabilities in Technology Production. Scandinavian J. of Information Systems Archive 14 (2) 91 - 105

19. Winschiers, H \& Fendler, J (2007) Assumptions Considered Harmful The Need to Redefine Usability. In: Usability and Internationalization, Part I, HCII 2007 Ed N. Aykin; LNCS Springer-Verlag Berlin Heidelberg

20. Wright, P \& McCarthy, J (2008) Empathy and Experience in HCI. CHI 08 637-646

21.Zenani, N M \& Scheub, H. (1992) The world and the word: tales and observations from the Xhosa oral tradition. Univ of Wisconsin Pr. 